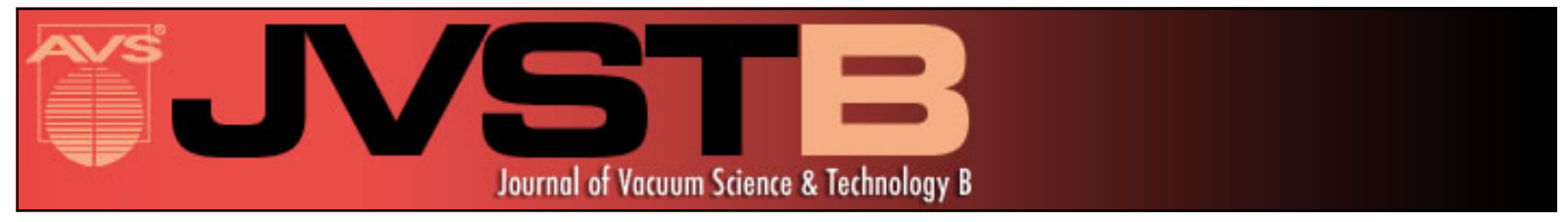

\title{
Excitation dependent photoluminescence measurements of the nonradiative lifetime and quantum efficiency in GaAs
}

S. R. Johnson, D. Ding, J.-B. Wang, S.-Q. Yu, and Y.-H. Zhang

Citation: Journal of Vacuum Science \& Technology B 25, 1077 (2007); doi: 10.1116/1.2720864

View online: http://dx.doi.org/10.1116/1.2720864

View Table of Contents: http://scitation.aip.org/content/avs/journal/jvstb/25/3?ver=pdfcov

Published by the AVS: Science \& Technology of Materials, Interfaces, and Processing

\section{Articles you may be interested in}

Strong excitation intensity dependence of the photoluminescence line shape in GaAs1-xBix single quantum well samples

J. Appl. Phys. 113, 144308 (2013); 10.1063/1.4801429

Optically pumped lasing from a single pillar microcavity with InGaAs/GaAs quantum well potential fluctuation quantum dots

J. Appl. Phys. 105, 053513 (2009); 10.1063/1.3074364

Time-resolved probing of the Purcell effect for InAs quantum boxes in GaAs microdisks

Appl. Phys. Lett. 78, 2828 (2001); 10.1063/1.1370123

Temperature and emission angle dependence of photoluminescence from an $\ln \mathrm{GaAs} / \mathrm{GaAs}$ quantum well in a microcavity structure

J. Appl. Phys. 83, 2282 (1998); 10.1063/1.366969

Intersubband photoluminescence of GaAs quantum wells under selective interband excitation Appl. Phys. Lett. 71, 1183 (1997); 10.1063/1.119619

\section{SHIMADZU Powerful, Multi-functional UV-Vis-NIR and Excellence in Science FTIR Spectrophotometers}

Providing the utmost in sensitivity, accuracy and resolution for applications in materials characterization and nano research

- Photovoltaics

- Polymers

- Thin films

- Paints

- Ceramics

- DNA film structures

- Coatings

- Packaging materials

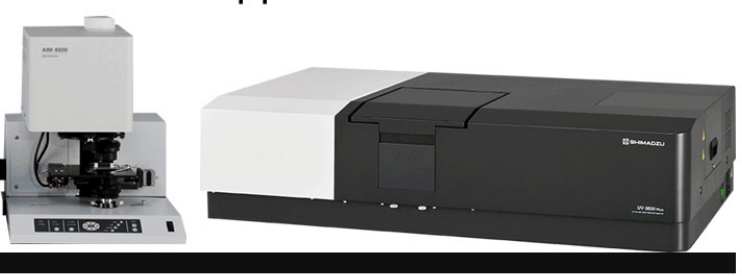




\title{
Excitation dependent photoluminescence measurements of the nonradiative lifetime and quantum efficiency in GaAs
}

\author{
S. R. Johnson, ${ }^{\text {a) }}$ D. Ding, J.-B. Wang, S.-Q. Yu, and Y.-H. Zhang \\ Center for Solid State Electronics Research, Arizona State University, Tempe, Arizona 85287-6206 \\ and Department of Electrical Engineering, Arizona State University, Tempe, Arizona 85287-6206
}

(Received 6 November 2006; accepted 5 March 2007; published 31 May 2007)

\begin{abstract}
The nonradiative lifetime and spontaneous emission quantum efficiency in molecular-beam epitaxy grown bulk GaAs is determined using injection level dependent photoluminescence (PL) measurements. These measurements were performed at temperatures of $300,230,100$, and $50 \mathrm{~K}$ using a HeNe pump laser with powers ranging from 0.3 to $40 \mathrm{~mW}$. The quantum efficiency and lifetime is inferred from the power law relation linking pump power and integrated PL signal that is predicted by the rate equations. The nonradiative lifetime for bulk GaAs is determined to be $0.3 \mu \mathrm{s}$, with an additional temperature dependent component attributed to the AlGaAs barriers that rapidly reduces the nonradiative lifetime at temperatures above $230 \mathrm{~K}$. The peak quantum efficiency is $>0.96$ at $300 \mathrm{~K}$ and $>0.99$ at temperatures below $230 \mathrm{~K}$. (C) 2007 American Vacuum Society. [DOI: 10.1116/1.2720864]
\end{abstract}

\section{INTRODUCTION}

Near unity quantum efficiency is critical to the performance of many luminescence based III-V devices. In order to grow these high quality materials by molecular-beam epitaxy (MBE) it is necessary to establish routine, straightforward, and accurate measurement techniques for the material quantum efficiency to feedback to the growth. Although substantial efforts have been devoted to such measurements, ${ }^{1-4}$ there are few straightforward methods that offer reliable data. In this work, an unambiguous experimental approach is presented for the determination of the nonradiative lifetime and the injection dependent spontaneous emission quantum efficiency that is based on the power law relationship between the various types of recombination found in III-V semiconductors.

In typical photoluminescence (PL) measurements the pump power $P_{\mathrm{PL}}(\mathrm{mW})$ that is absorbed in the active region, is proportional to the electron-hole pair photoexcitation density $\left(\mathrm{cm}^{-3} \mathrm{~s}^{-1}\right)$, which is equal to the total electron-hole pair recombination rate within the active region. Furthermore, the PL signal integrated over energy, $L_{\mathrm{PL}}$ (photons/s), at the photodetector is proportional to the spontaneous emission rate per unit area per unit length $\left(\mathrm{cm}^{-3} \mathrm{~s}^{-1}\right)$ from the active region. These relationships are described by

$$
P_{\mathrm{PL}}=c_{a}\left[A n+\left(1-\gamma_{r}\right) B n^{2}+C n^{3}\right], \quad L_{\mathrm{PL}}=c_{b} B n^{2},
$$

where $\gamma_{r}$ is the fraction of the spontaneous emission that is reabsorbed by the active region, ${ }^{5}$ and $c_{a}$ and $c_{b}$ are constants of proportionality that are determined by sample and measurement geometry. Here the recombination rates are written in terms of powers of the electron-hole concentration $n$, that physically reflect the various recombination processes that the electron-hole population undergoes. These processes are designated as Shockley-Read-Hall (SRH) recombination for

\footnotetext{
${ }^{a}$ Author to whom correspondence should be addressed; electronic mail:
} shane.johnson@asu.edu $n$, radiative recombination for $n^{2}$, and Auger recombination for $n^{3}$, with leading coefficients $A, B$, and $C$, respectively, ${ }^{6}$ which are valid under low injection, $n^{2}<N_{c} N_{v}$, where $N_{c}$ and $N_{v}$ are the effective density of states for the electrons and holes.

The assumptions implied in Eq. (1) are that the fraction of the spontaneous emission recycled and the fraction of the pump power that photoexcites electron-hole pairs are independent of injection level, which are, respectively, valid under low injection and when the pump photon energy is substantially larger than the active material bandgap. It is important to include the photon recycling factor $\gamma_{r}$ because it substantially affects the electron-hole density and the quantum efficiency. Each time a spontaneously emitted photon is recycled through band to band absorption, an electron-hole pair is generated which can be captured by either SRH or Auger recombination rather than emitting a photon, effectively increasing the nonradiative recombination by a factor of $\left(1-\gamma_{r}\right)^{-1}$.

From Eq. (1) the pump power can be written as powers of the PL signal with a leading coefficient for each of the recombination processes denoted as $A_{\mathrm{PL}}, B_{\mathrm{PL}}$, and $C_{\mathrm{PL}}$, for the $\mathrm{SRH}$, radiative, and Auger, respectively, with

$$
P_{\mathrm{PL}}=A_{\mathrm{PL}}\left(L_{\mathrm{PL}}\right)^{1 / 2}+B_{\mathrm{PL}} L_{\mathrm{PL}}+C_{\mathrm{PL}}\left(L_{\mathrm{PL}}\right)^{3 / 2} .
$$

The constants $A_{\mathrm{PL}}, B_{\mathrm{PL}}$, and $C_{\mathrm{PL}}$ are the best fit parameters determined by fitting Eq. (2) to pump power versus PL intensity measurements, from which the SRH and Auger coefficients are obtained by noting that

$$
\begin{aligned}
A= & A_{\mathrm{PL}} \frac{1}{\sqrt{c_{a}}}\left(\frac{B\left(1-\gamma_{r}\right)}{B_{\mathrm{PL}}}\right)^{1 / 2}, \\
& C=C_{\mathrm{PL}} \sqrt{c_{a}}\left(\frac{B\left(1-\gamma_{r}\right)}{B_{\mathrm{PL}}}\right)^{3 / 2}, \quad c_{b}=c_{a}\left(1-\gamma_{r}\right) / B_{\mathrm{PL}},
\end{aligned}
$$

where $B, c_{a}$, and $\gamma_{r}$ are straightforward to calculate. $B$ can be inferred from the thermal radiation [see Eqs. (6) $-(8)$ ]; $c_{a}$ is a 
function of photoexcited active volume and the fraction of pump power actively absorbed [see Eq. (9)]; and $\gamma_{r}$ is the fraction of spontaneous emission reabsorbed in the active region, which is determined via ray tracing over all solid angles. On the other hand, the luminescence constant of proportionality, $c_{b}$, is extremely difficult to determine, as it depends on sample emittance, absolute throughput of the spectrometer, absolute responsivity of the detector, and the collection efficiency of the sample luminescence, each of which are generally not accurately known. Fortunately, $c_{b}$ can be written in terms of $c_{a}$ [see Eq. (3)] and hence is eliminated from the calculation of the nonradiative coefficients.

A good figure of merit for the assessment of material quality, that is independent of sample geometry and accessible via PL measurements, is the spontaneous emission quantum efficiency $\eta_{q}$, which is defined as

$$
\begin{aligned}
\eta_{q} & \equiv \frac{B n^{2}}{A n+B n^{2}+C n^{3}} \\
& =\left(1+\frac{A_{\mathrm{PL}}}{B_{\mathrm{PL}}}\left(1-\gamma_{r}\right)\left(L_{\mathrm{PL}}\right)^{-1 / 2}+\frac{C_{\mathrm{PL}}}{B_{\mathrm{PL}}}\left(1-\gamma_{r}\right)\left(L_{\mathrm{PL}}\right)^{1 / 2}\right)^{-1},
\end{aligned}
$$

and in the second equation is written in terms of the integrated PL intensity and other experimental parameters. From the Boltzmann approximation (valid under low injection) the injection level (i.e., bandgap energy $E_{g}$ less quasi-Fermi level separation $\Delta F$ ) in terms of the electron-hole concentration is given by

$$
\frac{E_{g}-\Delta F}{k T}=\ln \left(\frac{N_{c} N_{v}}{n^{2}}\right)=\ln \left(\frac{c_{a}\left(1-\eta_{q} \gamma_{r}\right) B N_{c} N_{v}}{\eta_{q} P_{\mathrm{PL}}}\right),
$$

and is expressed in terms of pump power and $\eta_{q}$ on the right hand side, which is obtained by solving both the left hand equation in Eq. (1) and the left hand equation in Eq. (4) for $A n+B n^{2}+C n^{3}$, equating the two results, and solving the ensuing equation for $n^{2}$.

The radiative coefficient $B$ is a measure of the efficacy of the spontaneous emission from a semiconductor material, and its underlying fundamental properties are governed by thermodynamics, which ultimately establishes the light emission properties through the density of photon states and the photon occupation of those states. Therefore, it is straightforward to calculate $B n_{i}^{2}$ (where $n_{i}$ is the intrinsic, thermal equilibrium, electron-hole pair population), by noting that it is equivalent to the internal blackbody emission rate per unit area per unit length integrated over photon energies $h \nu$ above the bandgap energy, with

$$
\begin{aligned}
B n_{i}^{2} & =\frac{8 \pi c}{(h c)^{3}} \int_{E_{g}}^{\infty} \alpha_{o}(h \nu)\left(n_{o}(h \nu)\right)^{2} \frac{(h \nu)^{2} d h \nu}{e^{h \nu / k T}-1} \\
& \approx \frac{8 \pi c}{(h c)^{3}} \alpha_{g} n_{g}^{2}\left(E_{g}+k T\right)^{2} k T e^{-E_{g} / k T},
\end{aligned}
$$

where $n_{g}=n_{o}\left(E_{g}+k T\right)$ and $\alpha_{g}=\alpha_{o}\left(E_{g}+k T\right)$ are the index of refraction and absorption coefficient at the average spontane-

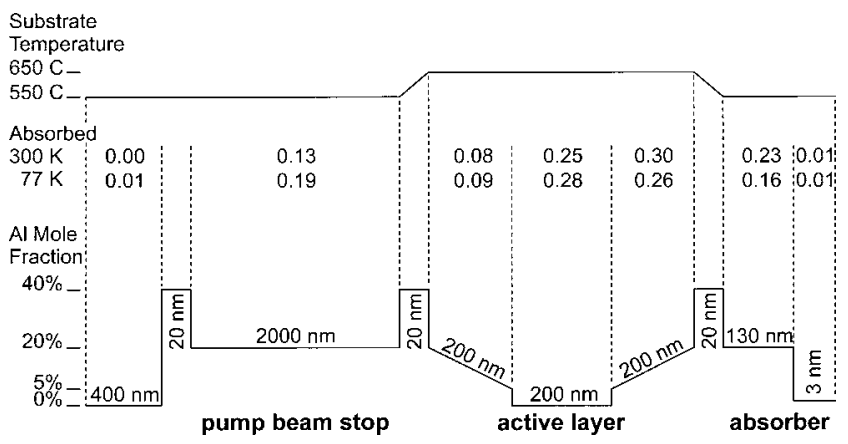

FIG. 1. Cross section (with sample surface on right hand side) and growth temperature profile of bulk GaAs sample (run B1657) used in photoluminescence measurements. The decimal numbers listed above each layer give the fraction of the pump radiation absorbed in that layer: upper for $300 \mathrm{~K}$ and lower for $77 \mathrm{~K}$.

ous emission energy $E_{g}+k T$. The intrinsic electron-hole concentration is accurately described by the Boltzmann approximation with

$$
n_{i}^{2}=N_{c} N_{v} e^{-E_{g} / k T}=32 \pi^{3}\left(m_{c} m_{v}\right)^{3 / 2}\left(\frac{m \cdot k T}{h^{2}}\right)^{3} e^{-E_{g} / k T},
$$

where $m$ is the electron mass, $m_{c}=m_{e}^{*} / m$ and $m_{v}=m_{h}^{*} / m$ are dimensionless, and $m_{e}^{*}$ and $m_{h}^{*}$ are the electron and hole effective masses, respectively. From Eqs. (6) and (7) the radiative coefficient is

$$
B \approx\left(\frac{1}{2 \pi}\right)^{2}\left(\frac{h}{m c}\right)^{3} \frac{n_{g}^{2} c \alpha_{g}}{\left(m_{c} m_{v}\right)^{3 / 2}}\left(\frac{E_{g}}{k T}+1\right)^{2},
$$

where the term $(h / m c)^{3}$ has units of volume and is the Compton wavelength $(2.43 \mathrm{pm})$ cubed. The term $n_{g}^{2}\left(m_{c} m_{v}\right)^{-3 / 2} c \alpha_{g}$ is a rate $\left(\sim 10^{18} \mathrm{~s}^{-1}\right)$ that is only weakly dependent on temperature and that is roughly constant across most direct band gap III-V semiconductors. Furthermore, the radiative coefficient goes by inverse temperature squared and band gap energy squared (which is weakly temperature dependent). From Eq. (8), the absorption coefficient and the band gap energy are key material figures of merit for spontaneous emission devices.

\section{EXPERIMENTAL RESULTS}

The GaAs/AlGaAs sample used in the PL measurements in this work was grown by MBE according to the cross section shown in Fig. 1, which is designed so that (i) the additional carriers generated in the graded $\mathrm{AlGaAs}$ layers are funneled into the active region increasing the injection level, (ii) the fraction of the pump power that contributes to the photoexcited electron-hole population is independent of temperature, and (iii) the pump radiation that passes through the active region is absorbed in a low temperature grown AlGaAs beam stop layer. All layers other than the active region are grown at low temperature to reduce their luminescence efficiency, minimizing pumping of the active region by spontaneous emission from other layers, particularly the $2000 \mathrm{~nm}$ thick beam stop layer; this was verified by confirming that no signal was present in the PL spectrum in the 
TABLE I. Summary of parameter values used in calculations.

\begin{tabular}{|c|c|c|c|}
\hline Parameter & Symbol & Value & Source \\
\hline GaAs absorption coefficient at $E_{g}+k T$ & $\alpha_{g}$ & $9050 \mathrm{~cm}^{-1}$ & Refs. 7 and 10 \\
\hline GaAs index of refraction at $E_{g}+k T$ & $n_{g}$ & 3.63 & Ref. 11 \\
\hline Dimensionless effective electron mass & $m_{c}^{g}$ & 0.067 & Ref. 6 \\
\hline Dimensionless effective hole mass & $m_{v}$ & 0.51 & Ref. 6 \\
\hline Photon recycling factor & $\gamma_{r}$ & 0.248 & Calculation \\
\hline GaAs combined density of states & $N_{c} N_{v}$ & $3.98 \times 10^{36}(T / 300)^{3} \mathrm{~cm}^{-6}$ & Ref. 6 \\
\hline GaAs zero temperature band gap & $E_{g}(0)$ & $1.519 \mathrm{eV}$ & Ref. 12 \\
\hline Einstein model coupling constant & $S_{g}$ & 5.982 & Ref. 8 \\
\hline Einstein temperature & $T_{E}^{b}$ & $262.9 \mathrm{~K}$ & Ref. 8 \\
\hline Pump energy per excitation event & $c_{a}$ & $1.99 \times 10^{-23} \mathrm{~mW} \mathrm{~s} \mathrm{~cm}^{3}$ & Eq. (9) \\
\hline Detector signal per generated photon & $c_{b}$ & $9.5 \times 10^{-23} \mathrm{~V} \mathrm{~s} \mathrm{~cm}^{3}$ & Eq. (3) \\
\hline GaAs Auger coefficient & $C$ & $7 \times 10^{-30} \mathrm{~cm}^{6} \mathrm{~s}^{-1}$ & Ref. 9 \\
\hline Radiation coefficient model constant & $B_{o}$ & $7.076 \times 10^{-11} \mathrm{~cm}^{3} \mathrm{~s}^{-1}$ & Fit \\
\hline Radiation coefficient model temperature & $T_{b}$ & $951.2 \mathrm{~K}$ & Fit \\
\hline
\end{tabular}

vicinity of the $\mathrm{Al}_{0.2} \mathrm{Ga}_{0.8} \mathrm{As}$ band gap energy. Temperature independent photoexcitation for a given pump power is accomplished by utilizing a $130 \mathrm{~nm}$ thick AlGaAs absorbing layer above the active region. Since the absorption coefficient of all layers is reduced at low temperature (the material band gap increases), the thickness of this layer is selected so that the additional pump radiation that reaches the active region at low temperature precisely compensates for the reduced absorption. A thicker (thinner) absorbing layer would result in the active region absorbing the largest fraction of pump radiation at low (room) temperature.

The fraction of the pump radiation that enters the sample and that is absorbed in each layer is given by the decimal number listed above each layer in Fig. 1, the upper number is for $300 \mathrm{~K}$ and the lower number is for $77 \mathrm{~K}$; although the fraction absorbed in each layer varies with temperature, the total fractional contribution to the GaAs active layer remains constant. The absorption lengths for the $633 \mathrm{~nm}$ HeNe pump radiation used in the design are $1 / \alpha_{\mathrm{GaAs}}(300 \mathrm{~K})=260 \mathrm{~nm}$, $1 / \alpha_{\mathrm{GaAs}}(77 \mathrm{~K})=300 \mathrm{~nm}, \quad 1 / \alpha_{\mathrm{AlGaAs}}(300 \mathrm{~K})=490 \mathrm{~nm}$, and $1 / \alpha_{\mathrm{AlGaAs}}(77 \mathrm{~K})=730 \mathrm{~nm}$. The room temperature values were taken from Ref. 7 and the low temperature values were estimated from the room temperature values using the shift in band gap energy (and hence absorption spectrum) with temperature. The linearly graded AlGaAs layers are grown at a constant growth rate of $16.0 \mathrm{~nm} / \mathrm{min}$, with the GaAs (AlAs) growth rate linearly varied from $12.8 \mathrm{~nm} / \mathrm{min}$ $(3.2 \mathrm{~nm} / \mathrm{min})$ at the $20 \% \mathrm{Al}$ mole fraction to $15.2 \mathrm{~nm} / \mathrm{min}$ $(0.8 \mathrm{~nm} / \mathrm{min})$ at the $5 \%$ mole fraction. The $\mathrm{Al}_{0.4} \mathrm{Ga}_{0.6} \mathrm{As}$ layers are transparent to the pump radiation and serve as barriers to prevent carrier spillage from one region of the sample to another.

Although not absolutely necessary, it is convenient to design the sample so that the photoexcitation density is independent of sample temperature, thus making the constant $c_{a}$ (pump power/photoexcitation density) independent of temperature, with

$$
c_{a}=\frac{V_{a} h \nu_{\mathrm{HeNe}}}{T_{\mathrm{HeNe}}\left(1-R_{\mathrm{HeNe}}\right) A_{\mathrm{HeNe}}},
$$

where $T_{\mathrm{HeNe}}=0.739$ is the measured fraction of the HeNe pump power that is transmitted through the optical system into the cryostat and onto the sample; $1-R_{\mathrm{HeNe}}=0.65$ is the fraction of $T_{\mathrm{HeNe}}$ that enters the sample (calculated using the optical constants in Ref. 7 and which changes $<1 \%$ over the temperatures in this work); and $A_{\mathrm{HeNe}}=0.63$ is the calculated fraction of $T_{\mathrm{HeNe}}\left(1-R_{\mathrm{HeNe}}\right)$ that is actively absorbed (which as mentioned above is roughly independent of temperature by design). The active volume of the photoexcited electronhole population is $V_{a}=\pi\left(D_{\mathrm{HeNe}} / 2\right)^{2} d_{a}=1.92 \times 10^{-8} \mathrm{~cm}^{3}$, where $D_{\mathrm{HeNe}}=350 \mu \mathrm{m}$ is the pump spot diameter and $d_{a}$ $=200 \mathrm{~nm}$ is the bulk GaAs layer thickness. The pump photon energy is $h \nu_{\text {in }}=3.14 \times 10^{-16} \mathrm{~mJ}$ (or $\mathrm{mW} \mathrm{s}$ ). Resulting in $c_{a}$ $=1.99 \times 10^{-23} \mathrm{~cm}^{3} \mathrm{~mW} \mathrm{~s}$, this and other parameters used in the calculations in this paper are listed in Table I.

Pump power dependent PL measurements of bulk GaAs are performed on the MBE grown (run B1657) sample using a HeNe laser with pump powers $P_{\mathrm{PL}}$ ranging from 0.3 to $40 \mathrm{~mW}$ at temperatures $T=300,230,100$, and $50 \mathrm{~K}$. These results are plotted in terms of pump power versus integrated PL signal in Fig. 2; the solid curves are fits of Eq. (2) to the data. Note that the injection level is not large enough to observe the 3/2 Auger power law dependence in the data, as $C n / B\left(1-\gamma_{r}\right) \ll 1$; therefore, the coefficient $C_{\mathrm{PL}}$ is negligible and cannot be accurately determined from these measurements. The best fit parameters $A_{\mathrm{PL}}$ and $B_{\mathrm{PL}}$ are given in Table II. As the injection increases in Fig. 2, the radiative lifetime decreases, resulting in the convergence of the curves toward the unity quantum efficiency line. The curves also move toward this line as temperature decreases due to an increasing radiative coefficient. Furthermore, if the injection level was increased further the experimental curves will eventually move away from the unity quantum efficiency line as Auger recombination becomes the dominant process. 


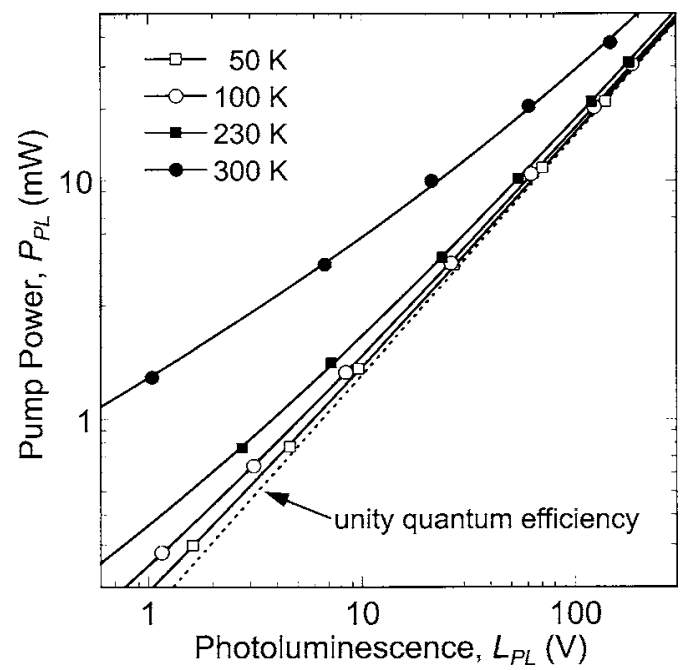

FIG. 2. Pump power vs PL signal from bulk GaAs at various temperatures. The solid curves are fits of a power law model to the data [see Eq. (2)].

The PL signal $L_{\mathrm{PL}}$ is obtained by integrating the PL spectrum (in units of detector output signal per unit wavelength) over wavelength, following the normalization of the spectrum for any wavelength dependent variations in the optical throughput of the monochromator-detector system, which is useful when comparing signals at different sample temperatures because the emission wavelength changes with temperature. This result is then normalized by multiplying it by the relative changes in the inverse of the monochromator slit width squared, i.e., $\times 1 / 4$ for $(0.5 \mathrm{~mm} / 1.0 \mathrm{~mm})^{2}$; this compensates for changes in the optical throughput of the monochromator due to changes in slit width (spectral resolution), which is typically reduced when sample temperature is reduced to avoid detector saturation from the larger low temperature luminescence intensities. The spectral resolution is $1.0 \mathrm{~nm}$ when the input and output slit widths are $1.0 \mathrm{~mm}$. The spectral resolution was more than adequate to resolve the PL line shape for all slit widths used. Attentive tracking and adjustment for optical throughput variations result in an approximately constant value of $c_{b}=c_{a}\left(1-\gamma_{r}\right) / B_{\mathrm{PL}}=9.5$ $\times 10^{-23} \mathrm{~V} \mathrm{~s} \mathrm{~cm}^{3}$.

\section{DISCUSSION}

Using a physical model, namely, the Einstein single oscillator photon occupation model, to describe the temperature

TABLE II. Experimental fitting parameters, SRH coefficients ascertained using fitting parameters, and calculated radiative coefficients.

\begin{tabular}{ccccc}
\hline \hline Temperature $(\mathrm{K})$ & $A_{\mathrm{PL}}\left(\mathrm{mW} / \mathrm{V}^{1 / 2}\right)$ & $B_{\mathrm{PL}}(\mathrm{mW} / \mathrm{V})$ & $A\left(\mathrm{~s}^{-1}\right)$ & $B\left(\mathrm{~cm}^{3} \mathrm{~s}^{-1}\right)$ \\
\hline 50 & 0.0381 & 0.1543 & $3.01 \times 10^{6}$ & $2.55 \times 10^{-8}$ \\
100 & 0.0886 & 0.1571 & $3.46 \times 10^{6}$ & $6.33 \times 10^{-9}$ \\
230 & 0.2007 & 0.1606 & $3.29 \times 10^{6}$ & $1.14 \times 10^{-9}$ \\
300 & 1.3396 & 0.1562 & $1.67 \times 10^{7}$ & $6.41 \times 10^{-10}$ \\
\hline
\end{tabular}

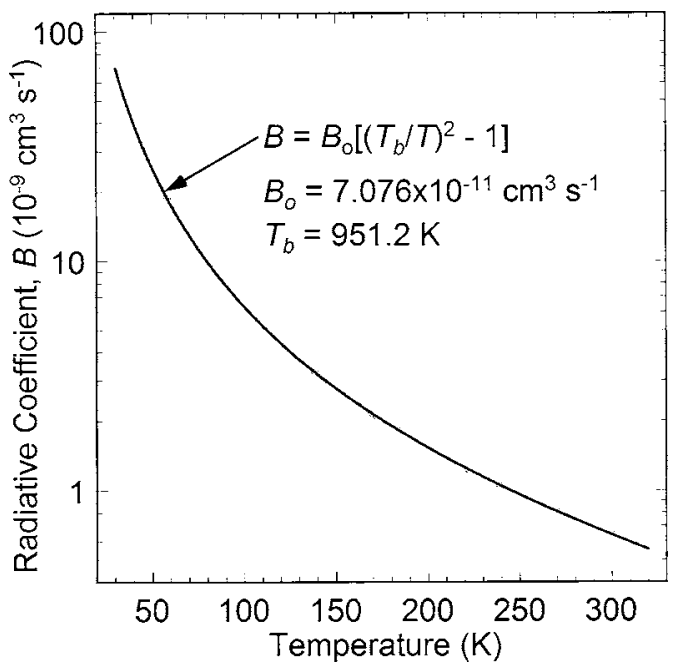

FIG. 3. Radiative coefficient vs temperature for GaAs; the solid gray curve is a calculation using Eq. (10) and the solid black curve is a fit of the model shown in the plot to the calculated curve over the 30 to $320 \mathrm{~K}$ temperature range.

dependence of the band gap energy, the radiative coefficient given in Eq. (8) is explicitly written in terms of temperature as

$$
\begin{aligned}
B & \approx\left(\frac{1}{2 \pi}\right)^{2}\left(\frac{h}{m c}\right)^{3} \frac{n_{g}^{2} c \alpha_{g}}{\left(m_{c} m_{v}\right)^{3 / 2}}\left(\frac{E_{g}(0)}{k T}-\frac{S_{g} T_{E} / T}{e^{T_{E} / T}-1}+1\right)^{2} \\
& \approx B_{o}\left(\left(\frac{T_{b}}{T}\right)^{2}-1\right)
\end{aligned}
$$

where $E_{g}(0)$ is the zero temperature band gap, $S_{g}$ is a dimensionless coupling constant, and $T_{E}$ is the Einstein temperature where $k T_{E} /\left(e^{T_{E} / T}-1\right)$ is the average thermal energy of the phonon population. ${ }^{8}$ The radiative coefficient calculated using Eq. (10) is shown in Fig. 3 as a solid gray curve and the parameters used in the calculation are listed in Table I. The specific temperature dependence of the radiative coefficient over the temperature range of interest is determined by fitting an empirical two parameter model [see right hand equation in Eq. (10)] to the calculated values, which is shown as the solid black curve that nicely overlaps the calculated curve in Fig. 3. The best fit parameters over the $30-320 \mathrm{~K}$ temperature range are $B_{o}=7.076 \times 10^{-11} \mathrm{~cm}^{3} \mathrm{~s}^{-1}$ and $T_{b}=951.2 \mathrm{~K}$. The values of the radiative coefficient, which vary by a factor of 40 over the temperature range of the measurements, are given in Table II; this large variation is a major contributor to the improvement in quantum efficiency at low temperature.

The SRH coefficients $A$ are calculated using Eq. (3) and are given in Table II. The spontaneous emission quantum efficiency $\eta_{q}$ is calculated using the fitting parameters $A_{\mathrm{PL}}$ and $B_{\mathrm{PL}}$, Eq. (4), and the integrated PL signal $L_{\mathrm{PL}}$ and is plotted against injection level, $\left(E_{g}-\Delta F\right) / k T$, in Fig. 4; $\left(E_{g}\right.$ $-\Delta F) / k T$ is calculated using Eq. (5) and the pump power $P_{\mathrm{PL}}$. The solid curves are fits of the model, 


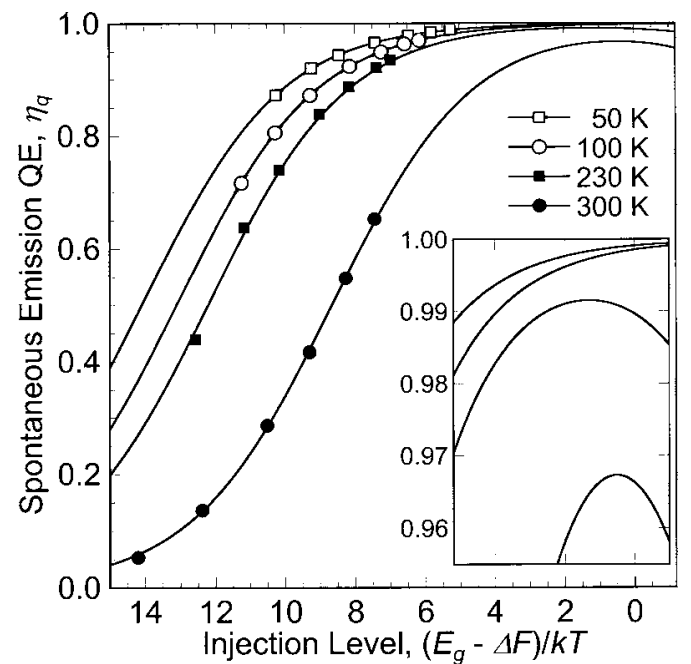

FIG. 4. Spontaneous emission quantum efficiency (QE) vs injection level; the peak quantum efficiency region is shown in the inset. The solid curves are fits of Eq. (11) to the data.

$$
\eta_{q}=\left(1+\frac{A}{B} \sqrt{\frac{e^{\left(E_{g}-\Delta F\right) / k T}}{N_{c} N_{v}}}+\frac{C}{B} \sqrt{\frac{N_{c} N_{v}}{e^{\left(E_{g}-\Delta F\right) / k T}}}\right)^{-1},
$$

to the data, where $A$ is a fitting parameter and $C=7$ $\times 10^{-30} \mathrm{~cm}^{6} \mathrm{~s}^{-1}$ is fixed at a recent value given in the literature. ${ }^{9}$ The values for $A$ obtained from these fits are within $1 \%$ of those calculated using Eq. (3). The peak quantum efficiency region is shown in the inset of Fig. 4. It should be noted that in this analysis $\left(E_{g}-\Delta F\right) / k T$ is calculated from both $L_{\mathrm{PL}}$ and $P_{\mathrm{PL}}$ since $\eta_{q}$ appears in Eq. (5); to write $\left(E_{g}-\Delta F\right) / k T$ strictly in terms of $P_{\mathrm{PL}}$ the cubic relation given in Eq. (1) must be used, which in general results in a much more complex expression. However, since $C_{\mathrm{PL}}$ does not appear in the present measurements, the injection level in terms of $P_{\mathrm{PL}}$ alone can be written in relatively simple terms as

$$
\begin{aligned}
\frac{E_{g}-\Delta F}{k T} & =\ln \left(\frac{N_{c} N_{v}}{n^{2}}\right) \\
& =\ln \left(\frac{4 B_{\mathrm{PL}}}{A_{\mathrm{PL}}^{2}} \frac{c_{a}\left(1-\gamma_{r}\right) B N_{c} N_{v}}{\left(\sqrt{1+\left(4 B_{\mathrm{PL}} / A_{\mathrm{PL}}^{2}\right) P_{\mathrm{PL}}-1}\right)^{2}}\right) .
\end{aligned}
$$

The injection levels obtained from Eq. (12) are essentially the same as those obtained from Eq. (5).

The experimentally determined SRH coefficient as a function of sample temperature is plotted in Fig. 5, where the solid blue curve is a fit of an Arrhenius model to the data; the model is given in the plot. This data indicates that there are two components to the SRH coefficient (or nonradiative lifetime): (i) a constant component $A_{0}=3.2 \times 10^{6} \mathrm{~s}^{-1} \quad\left(1 / A_{0}\right.$ $=310 \mathrm{~ns}$ ) that most likely originates from deep levels (traps) in the bulk GaAs active layer and (ii) a temperature dependent component $A_{a} e^{-E_{a} / k T}$ with frequency $A_{a}=4.9 \times 10^{14} \mathrm{~s}^{-1}$ and characteristic activation energy $E_{a}=450 \mathrm{meV}$ that rapidly increases near room temperature and that most likely originates from nonradiative recombination in the AlGaAs barriers.

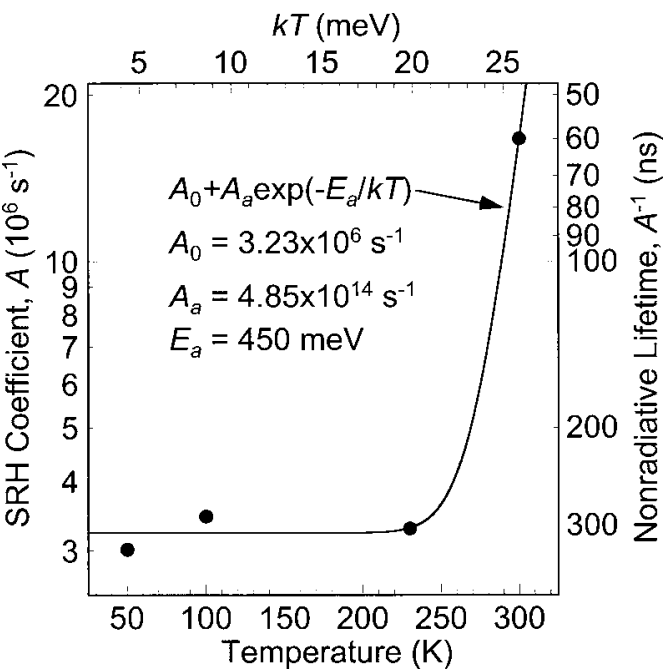

FIG. 5. SRH coefficient vs sample temperature; the solid curve is a fit to the data of the model shown in the plot.

The sample design and measurement approach presented here makes the analysis straightforward. However, it is not absolutely necessary, since the above spontaneous emission quantum efficiency and lifetime measurements can be performed on most luminescence samples, where the constant of proportionality $c_{a}$ may have to be determined separately for each temperature and the fitting constant $B_{\mathrm{PL}}$ may differ for each temperature if variations in the optical throughput are not monitored and corrected for. Furthermore, if sufficient pump power is available, higher injection can be achieved without funneling additional carriers into the active layer, eliminating the need for the graded AlGaAs layers. The beam stop layer can also be omitted provided a very thin GaAs buffer $(\sim 20 \mathrm{~nm})$ is grown on a semi-insulating substrate so that an insignificant amount of luminescence is generated in the buffer compared to the active region. Moreover, it is best to minimize photon recycling since its effect must be calculated and hence can add uncertainty to the determination of the nonradiative coefficients. The photon recycling factor $\gamma_{r}$ can be as small as 0.1 for thin (quantum well) active layers grown on semi-insulating substrates which parasitically absorb spontaneous emission without any further emission that can be reabsorbed by the active region. The definition and role of the photon recycling factor is further discussed in Ref. 5.

\section{CONCLUSIONS}

The nonradiative lifetime and spontaneous emission quantum efficiency of bulk GaAs is determined using temperature and excitation density dependent photoluminescence. The quantum efficiency is inferred from the power law relation between integrated PL intensity and pump power, where the PL intensity increases as the square of the pump power when SRH recombination dominates, linearly when the quantum efficiency is unity, and sublinearly when Auger recombination dominates. At temperatures below $230 \mathrm{~K}$ the nonradiative lifetime of bulk GaAs is $310 \mathrm{~ns}$, while at temperatures 
above $230 \mathrm{~K}$ the nonradiative lifetime rapidly decreases to $60 \mathrm{~ns}$ at $300 \mathrm{~K}$. The temperature dependent part of the nonradiative lifetime is attributed to deep levels in the AlGaAs barriers. The peak spontaneous emission quantum efficiency is determined to be $>0.96$ at $300 \mathrm{~K}$ and $>0.99$ at temperatures below $230 \mathrm{~K}$.

\section{ACKNOWLEDGMENT}

This work is supported by a MURI program from the Air Force Office of Scientific Research Grant No. FA9550-04-10374.

${ }^{1}$ T. Fleck, M. Schmidt, and C. Klingshirn, Phys. Status Solidi A 198, 248 (2003).
${ }^{2}$ R. Westphaling, P. Ullrich, J. Hoffmann, H. Kalt, C. Klingshirn, K. Ohkawa, and D. Hommel, J. Appl. Phys. 84, 6871 (1998).

${ }^{3}$ R. K. Ahrenkiel, Solid-State Electron. 35, 239 (1992).

${ }^{4}$ I. Schnitzer, E. Yablonovitch, C. Caneau, and T. J. Gmitter, Appl. Phys. Lett. 62, 131 (1993).

${ }^{5}$ J.-B. Wang, S. R. Johnson, D. Ding, S.-Q. Yu, and Y.-H. Zhang, J. Appl. Phys. 100, 043502 (2006).

${ }^{6}$ S. L. Chuang, Physics of Optoelectronic Devices (Wiley, New York, 1995), Chaps. 2 and 10.

${ }^{7}$ E. D. Palik, Handbook of Optical Constants of Solids (Academic, New York, 1985).

${ }^{8}$ S. R. Johnson and T. Tiedje, J. Appl. Phys. 78, 5609 (1995).

${ }^{9}$ U. Strauss, W. W. Ruhle, and K. Kohler, Appl. Phys. Lett. 62, 55 (1993).

${ }^{10}$ M. D. Sturge, Phys. Rev. 127, 768 (1962).

${ }^{11}$ J. S. Blakemore, J. Appl. Phys. 53, R123 (1982).

${ }^{12}$ D. D. Sell, Phys. Rev. B 6, 3750 (1972). 